\title{
Compliance alla terapia dietetica
}

\author{
C. D’Alessandro, E. Colombini, G. Pasquariello, G. Sbragia, A. Cupisti
}

Divisione di Nefrologia, Dipartimento di Medicina Interna, Università di Pisa, Pisa

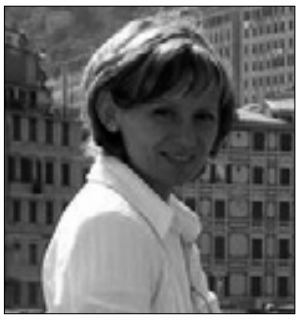

Claudia D'Alessandro

\section{Caso clinico 1}

Maschio di 53 anni giunge alla nostra osservazione nell'ottobre 2009 per insufficienza renale cronica, calo ponderale di circa $5 \mathrm{~kg}$ nell'ultimo mese (dopo l'inizio di una "dieta" ipoproteica) con astenia.

Dall'anamnesi: a 29 anni trauma renale sinistro con macroematuria. A 32 anni, diagnosi di sclerosi multipla; a 34 anni ulcera duodenale. Nel 2006 riscontro di policitemia vera e di un lieve aumento dei livelli di creatininemia. Nel 2009 primo riscontro di ipertensione arteriosa $(160 / 100 \mathrm{mmHg})$ e peggioramento della funzione renale (clearance della creatinina di $32 \mathrm{ml} / \mathrm{min}$ ). L'ecografia dell'addome evidenzia reni ai limiti della norma con spessore parenchimale nettamente ridotto e scarsa differenziazione cortico-midollare. Al paziente viene consigliata terapia anti ipertensiva con ramipril $5 \mathrm{mg}$ /die e una "dieta ipoproteica con assunzione di pasta aproteica".

La Tabella I riporta i principali parametri in occasione della prima visita. E.O.: non edema periferico, peso corporeo $70 \mathrm{~kg}$, SGA- B. P.A. $120 / 75 \mathrm{mmHg}$. Si rilevano inoltre: G.B. $6000 / \mathrm{mm}^{3}$, G.R. $6.600 .000 / \mathrm{mm}^{3}$, Ematocrito $58 \%, \mathrm{Hb} 19,4 \mathrm{~g} / \mathrm{dL}$, Piastrine $167.000 / \mathrm{mm}^{3}$; esame urine: $\mathrm{pH} 5,5$, densità 1012 , proteinuria assente, sedimento n.d.n.

Il paziente viene sottoposto a un counselling dietetico per modificare la terapia dietetica (ipoproteica $0,6 \mathrm{~g} /$ $\mathrm{kg} /$ die, ipofosforica $8 \mathrm{mg} / \mathrm{kg} /$ die, iposodica $2-3 \mathrm{~g} / \mathrm{die}$, normocalorica $30 \mathrm{kcal} / \mathrm{kg}$ peso ideale).

A distanza di un mese il paziente riferisce un miglioramento soggettivo con aumento del senso di benessere e del peso corporeo $(71 \mathrm{~kg})$. Si rileva una riduzione significativa dell'urea plasmatica, dell'urea urinaria e della sodiuria mentre i livelli di creatininemia risultano stabili
(Tab. I). PTH 113,7 pg/mL (non sono disponibili misurazioni precedenti).

A distanza di 12 mesi il paziente si presenta in buone condizioni generali, funzione renale residua stabile $\mathrm{e}$ buon compenso metabolico (Tab. I).

L'analisi bioimpedenziometrica mostra una buona composizione corporea con un angolo di fase di $5.6^{\circ}$ e un Body Cell Mass Index di 10,2 kg/m².

\section{Caso clinico 2}

Donna di 75 anni affetta da diabete mellito di tipo 2 da 15 anni, ipertensione arteriosa e insufficienza renale cronica, giunge presso il nostro ambulatorio nel febbraio 2008 per peggioramento dei valori di funzione renale $\mathrm{e}$ malessere.

Dall'anamnesi: nel 1998 comparsa di dispnea da sforzo e riscontro di riduzione della funzione ventricolare sinistra (FE 43\%), ipertrofia ventricolare sinistra e stenosi subcritica della carotide interna sinistra. Nell'agosto 1999 resezione segmentaria del sigma per adenocarcinoma. Nell'agosto 2000 edema polmonare acuto e riscontro coronarografico di stenosi subcritiche coronariche. Nel 2007 riscontro di creatininemia di $1,6 \mathrm{mg} / \mathrm{dL}$ con eGFR calcolato $33,5 \mathrm{ml} / \mathrm{min} / 1,73 \mathrm{~m}^{2}$. Alla paziente viene consegnata una dieta per diabetici con $1,2 \mathrm{~g} / \mathrm{kg}$ p.c. di proteine.

Terapia farmacologica: atorvastatina $20 \mathrm{mg} / \mathrm{die}$, amiodarone $200 \mathrm{mg} / \mathrm{die}$, doxazosina $4 \mathrm{mg} / \mathrm{die}$, amlodipina $10 \mathrm{mg} / \mathrm{die}$, carvedilolo $50 \mathrm{mg} / \mathrm{die}$, lisina acetilsalicilato $1 /$ die, allopurinolo $150 \mathrm{mg} /$ die, candesartan $16 \mathrm{mg} /$ die, furosemide $125 \mathrm{mg} 3$ volte la settimana, nitroglicerina $10 \mathrm{mg} /$ die, repaglinide $6 \mathrm{mg} /$ die, metformina $1000 \mathrm{mg} /$ die.

La Tabella II riporta i principali parametri al tempo della prima visita. E.O.: lieve edema agli arti inferiori, peso corporeo $84 \mathrm{~kg}$, SGA-B. P.A. 150/95 mmHg. Esame urine: $\mathrm{pH} 5,5$, densità 1012 , proteinuria assente, sedimento n.d.n. 
Ecografia addome: epatosteatosi, dimensioni renali 11 $\mathrm{cm}$ bilateralmente con struttura diffusamente alterata. Viene sospesa terapia con metformina, iniziata terapia insulinica e prescritta una dieta ipoproteica $(0,6 \mathrm{~g} / \mathrm{kg}$ peso ideale/die), ipofosforica $(8 \mathrm{mg} / \mathrm{kg} / \mathrm{die})$, a contenuto controllato di sodio (2-3 g/die), lievemente ipocalorica (27 kcal/ kg peso ragionevole) a ridotto contenuto di zuccheri semplici, somministrata mediante counselling dietetico. Consigliato un aumento dell'attività fisica.

Dopo sei mesi dall'inizio del trattamento si rende necessaria la sospensione della statina per comparsa di mialgie. Inoltre, per un cattivo controllo glico-metabolico viene aumentata la posologia della repaglinide e introdotta

TABELLA I - CASO CLINICO 1: DATI RELATIVI ALLA PRIMA VISITA E DOPO UNO E DODICI MESI DALLINIZIO DELLA TERAPIA NUTRIZIONALE

\begin{tabular}{|l|c|c|c|}
\hline & Inizio & 1 mese & 12 mesi \\
\hline Creatininemia, mg/dL & 3,2 & 3,3 & 3,4 \\
\hline Urea pl., mg/dL & 82 & 40 & 45 \\
\hline Potassiemia, mEq/L & 5,7 & 5,6 & 5,3 \\
\hline Sodiemia, $\mathrm{mEq} / \mathrm{L}$ & 139 & 135 & 137 \\
\hline Calcemia, $\mathrm{mg} / \mathrm{dL}$ & 9,0 & 9,1 & 9,2 \\
\hline Fosforemia, mg/dL & 4,0 & 3,7 & 3,8 \\
\hline Uricemia, $\mathrm{mg} / \mathrm{dL}$ & 7,0 & 7,9 & 4,5 \\
\hline Urea urinaria, $\mathrm{g} / 24$ ore & 18,7 & 10 & 12,3 \\
\hline Sodiuria, $\mathrm{mEq} / 24$ ore & 201 & 117 & 134 \\
\hline nPCR, $\mathrm{g} / \mathrm{kg} / \mathrm{die}$ & 0,97 & 0,60 & 0,67 \\
\hline IMC, $\mathrm{kg} / \mathrm{m}^{2}$ & 21,8 & 22,2 & 22,8 \\
\hline
\end{tabular}

TABELLA II - CASO CLINICO 2: DATI RELATIVI ALLA PRIMA VISITA E DOPO SEI MESI E DOPO TRENTA MESI DALLINIZIO DELLA TERAPIA NUTRIZIONALE

\begin{tabular}{|l|c|c|c|}
\hline & Inizio & $\mathbf{6}$ mesi & 30 mesi \\
\hline Creatininemia, mg/dL & 2,5 & 1,9 & 1,5 \\
\hline Urea pl., mg/dL & 230 & 121 & 89 \\
\hline Potassiemia, mEq/L & 5,0 & 4,3 & 4,0 \\
\hline Sodiemia, mEq/L & 133 & 142 & 143 \\
\hline Calcemia, mg/dl & 9,0 & 9,8 & 9,5 \\
\hline Fosforemia, mg/dL & 5,0 & 4,2 & 4,2 \\
\hline Hb1Ac, $\%$ & 7,2 & 7.0 & 6.5 \\
\hline Colesterolo tot., mg/dL & 228 & 210 & 188 \\
\hline Colesterolo LDL, mg/dL & 140 & 133 & 100 \\
\hline Trigliceridi, $\mathrm{mg} / \mathrm{dL}$ & 320 & 245 & 210 \\
\hline Hb, $\mathrm{g} / \mathrm{dL}$ & 11,6 & 11,6 & 11,8 \\
\hline IMC, $\mathrm{kg} / \mathrm{m}^{2}$ & 30,4 & 29,0 & 28,5 \\
\hline
\end{tabular}

insulina Levemir alle ore 23. Si evidenzia una riduzione dei livelli di urea plasmatica e della creatininemia e un miglioramento del profilo lipidico (Tab. II). PA 150/70 mmHg.

A distanza di 30 mesi si rileva un ulteriore miglioramento della funzione renale e del profilo glico-metabolico (Tab. II). Pressione arteriosa 130/70 mmHg.

La paziente riferisce un miglioramento soggettivo. Il BMI attualmente è $28,5 \mathrm{~kg} / \mathrm{m}^{2}$ e l'analisi bioimpedenziometrica rileva un angolo di fase di $4,5^{\circ}$, un BCMI di 8 $\mathrm{kg} / \mathrm{m}^{2}$. La paziente continua a seguire la terapia dietetica e abbiamo consigliato di aumentare l'attività motoria in modo da migliorare la composizione corporea a favore della massa magra.

\section{Discussione}

La terapia nutrizionale è uno dei cardini della terapia conservativa dell'Insufficienza Renale Cronica con o senza diabete. Essa è in grado di prevenire o correggere segni, sintomi e complicanze dell'insufficienza renale, di procrastinare l'inizio della dialisi e di mantenere lo stato nutrizionale $(1,2)$. La dieta deve essere ridotta in proteine perché molte delle tossine e dei cataboliti ritenuti derivano dalle proteine esogene, e perché la restrizione proteica rappresenta una condizione necessaria, anche se non sufficiente, per la contestuale riduzione dell'apporto di sodio e fosforo che contribuisce agli effetti terapeutici; altra caratteristica fondamentale della terapia dietetica è l'adeguatezza energetica (3).

La fonte principale di calorie è costituita dagli alimenti artificiali aproteici, che per alcuni pazienti risultano però poco appetibili (4). Come a tutti noto, di recente si è anche aggiunto il problema, non certo trascurabile, della rimborsabilità di questi prodotti, il cui prezzo di mercato ha raggiunto cifre difficilmente sostenibili per molte persone, soprattutto le più anziane.

Nei due casi descritti si ritrovano problematiche di ordinaria pratica clinica e dietologica. Il primo caso è un paziente giovane, attivo, che deve sapersi organizzare anche quando è lontano da casa, e che ha perso peso a seguito di una dieta incongrua. Il secondo è quello di una donna di età più avanzata, diabetica in sovrappeso, casalinga, e che si occupa della preparazione dei pasti per tutta la famiglia.

Queste due situazioni diverse tra loro fanno capire che per avere una buona aderenza alla prescrizione dietetica è importante calarsi nella realtà quotidiana del paziente, comprenderne le dinamiche familiari, sociali, lavorative e fornire le soluzioni possibili (4). La disponibilità di personale motivato e qualificato è fondamentale al fine di 
garantire un sostegno al paziente nefropatico e ai familiari fornendo loro anche le informazioni corrette.

Il counselling dietetico che viene offerto ai nostri pazienti prevede una iniziale raccolta della storia dietetica e di informazioni relative allo stile di vita, all'organizzazione domestica (chi si occupa della spesa, di cucinare) e della giornata (attività lavorativa, attività motoria). Vengono poi spiegati alcuni aspetti della terapia dietetica nell'insufficienza renale, quali il controllo dell'apporto di sodio (sale aggiunto, sale contenuto negli alimenti e sale "di farmacia"), il controllo dell'apporto di fosforo (perché si escludono latte e formaggi, e prodotti con additivi), chiarimenti su alcuni luoghi comuni non sempre corretti ("la carne rossa è più proteica della carne bianca", "il bere tanto per far funzionare meglio i reni”); alla fine viene definito il piano alimentare. Nei casi descritti la terapia nutrizionale consisteva in una dieta ipoproteica $(0,6 \mathrm{~g} /$ $\mathrm{kg} / \mathrm{die}$ ) convenzionale, a contenuto controllato di sodio (2-3 g/die) corrispondente a un apporto di sale inferiore a $6 \mathrm{~g} / \mathrm{die}$, ipofosforica $(8 \mathrm{mg} / \mathrm{kg} / \mathrm{die})$, normocalorica $(30 \mathrm{kcal} / \mathrm{kg}$ peso ideale/die) nel primo caso, e lievemente ipocalorica (27 kcal/ $\mathrm{kg}$ peso ragionevole/die) con ridotto contenuto di zuccheri semplici nel secondo. Per quantificare l'apporto di calorie e proteine è stato utilizzato nel primo caso il peso ideale, calcolato come altezza $^{2}\left(\mathrm{~m}^{2}\right) \cdot 22\left(\mathrm{~kg} / \mathrm{m}^{2}\right)$ mentre nel secondo caso, dove il peso attuale della paziente si discostava in modo significativo dal peso ideale, abbiamo utilizzato un peso ragionevole calcolato come altezza ${ }^{2}\left(\mathrm{~m}^{2}\right) \cdot 25\left(\mathrm{~kg} / \mathrm{m}^{2}\right)$.

Il piano alimentare viene elaborato rispettando, per quanto possibile, i gusti e le necessità familiari, lavorative $o$ di svago dei pazienti grazie alle informazioni raccolte durante il colloquio. Ai pazienti viene richiesto un regolare controllo del peso corporeo, fondamentale soprattutto in presenza di terapia con diuretici, e un diario dietetico da compilare prima del primo controllo (a un mese).

Durante le visite il dietista provvede a una valutazione dello stato di nutrizione (5) mediante analisi bioimpedenziometrica e SGA e affronta, per risolvere, i problemi riferiti dal paziente.

La principale criticità di questo approccio che prevede il counselling dietetico personalizzato è proprio la carenza di dietisti dedicati alla nefrologia, già ampiamente documentata sia a livello nazionale che internazionale. Nel 2007 un'indagine condotta nell'ambito del progetto $\mathrm{Ne}$ phrontieres ha evidenziato che il $71,5 \%$ (7 Centri su 10) non dispone di un dietista. Un'indagine europea condotta dalla British Renal Society ha calcolato che servirebbe un dietista a tempo pieno ogni 180 pazienti affetti da insufficienza renale in stadio 4-5, traguardo difficilmente raggiungibile in qualsiasi Paese. Ad esempio, in Francia servirebbero circa 500 dietisti per i 32.000 pazienti con insufficienza renale avanzata in stadio terminale, ma $\mathrm{i}$ dietisti dedicati non sono più di 50 (6).

In conclusione, la terapia nutrizionale è presupposto irrinunciabile nel trattamento dei pazienti nefropatici, e la figura del dietista assume un ruolo centrale per una corretta gestione che possa garantire risultati e sicurezza.

\section{Riassunto}

La terapia nutrizionale è uno dei cardini della terapia conservativa dell'Insufficienza Renale Cronica (IRC). $\grave{E}$ in grado di contrastare segni, sintomi e complicanze dell'insufficienza renale, di procrastinare l'inizio della dialisi e di mantenere lo stato nutrizionale. La dieta deve essere ridotta in proteine perché molte delle tossine $\mathrm{e}$ dei cataboliti ritenuti derivano dalle proteine esogene, e perché la restrizione proteica rappresenta una condizione necessaria, anche se non sufficiente, per la contestuale riduzione dell'apporto di sodio e fosforo che contribuisce agli effetti terapeutici. Altra caratteristica fondamentale della terapia dietetica è l'adeguatezza energetica.

I due casi descritti rappresentano quello che spesso accade nella pratica clinica nel paziente con IRC cui viene prescritta una dieta ipoproteica, e sottolineano l'importanza del counselling dietetico per la sicurezza e l'efficacia della terapia nutrizionale nel paziente renale con o senza diabete mellito.

Parole chiave: Dieta ipoproteica, Insufficienza Renale Cronica, Counselling dietetico

Indirizzo degli Autori:

Claudia D'Alessandro, MD

Divisione di Nefrologia

Dipartimento di Medicina Interna

Università di Pisa, Pisa

dalex.c@virgilio.it 


\section{Bibliografia}

1. Mitch WE, Remuzzi G. Diets for patients with chronic kidney disease, still worth prescribing. J Am Soc Nephrol 2004; 15(1): 234-7.

2. Fouque D, Aparicio M. Eleven reasons to control the protein intake of patients with chronic kidney disease. Nat Clin Pract Nephrol 2007; 3: 383-92.

3. Cupisti A. Definizione della terapia nutrizionale nel trattamento conservativo del paziente con IRC. Giorn Ital Nefrol 2008; S42: S35-8.

4. Bellizzi V, Bedogni G, Quintaliani G. Compliance alla dieta nell'Insufficienza Renale Cronica (IRC). Giorn Ital Nefrol 2008; S42: S45-9.

5. D'Alessandro C, Morelli E, Cupisti A. Metodi di valutazione dello stato nutrizionale. Anthologica Medica Santoriana 2004; 1: 39-42.

6. Fouque D. Should we still prescribe a reduction in protein intake for CKD patients? Am J Nephrol 2006; 26 (Suppl 1): S8-9.

\section{TEST di VERIFICA - 1}

1) La dieta nell'insufficienza renale cronica deve avere le seguenti caratteristiche

a) Ipoproteica-normocalorica-ipofosforica

b) Ipoproteica-ipocalorica-normofosforica

c) Iperproteica- ipercalorica-normofosforica

d) Nessuna di queste

2) Metodiche utilizzate nella valutazione dello stato nutrizionale sono

a) Bio-Impedenziometria vettoriale

b) Albuminemia

c) Valutazione Globale Soggettiva (SGA)

d) Tutte queste

3) Nelle UU.OO. di Nefrologia e Dialisi Italiane, la prevalenza stimata di un dietista dedicato è:
a) $70 \%$
b) $50 \%$
c) $30 \%$
d) $20 \%$

Le risposte corrette alle domande sono a pag. 25 